\title{
Protocol: Precision engineering of plant gene loci by homologous recombination cloning in Escherichia coli Laura C Roden ${ }^{1,3}$, Berthold Göttgens ${ }^{2}$ and Effie S Mutasa-Göttgens*1
}

\author{
Address: ${ }^{1}$ Broom's Barn Research Station, Higham, Bury St Edmunds, Suffolk IP28 6NP, UK, ${ }^{2}$ Cambridge Institute for Medical Research, Hills \\ Road, Cambridge, CB2 2XY, UK and ${ }^{3}$ Dept. Mol. \& Cell Biol., UCT, Private Bag Rondebosch, 7701, Cape Town, South Africa \\ Email: Laura C Roden - LRODEN@science.uct.ac.za; Berthold Göttgens - bg200@cam.ac.uk; Effie S Mutasa- \\ Göttgens* - effie.mutasa@bbsrc.ac.uk \\ * Corresponding author
}

Published: 29 September 2005

Plant Methods 2005, I:6 doi:10.1 186/1746-48|1-1-6

This article is available from: http://www.plantmethods.com/content/l/1/6

(c) 2005 Roden et al; licensee BioMed Central Ltd.

This is an Open Access article distributed under the terms of the Creative Commons Attribution License (http://creativecommons.org/licenses/by/2.0), which permits unrestricted use, distribution, and reproduction in any medium, provided the original work is properly cited.
Received: 14 July 2005

Accepted: 29 September 2005

\begin{abstract}
Plant genome sequence data now provide opportunities to conduct molecular genetic studies at the level of the whole gene locus and above. Such studies will be greatly facilitated by adopting and developing further the new generation of genetic engineering tools, based on homologous recombination cloning in Escherichia coli, which are free from the constraints imposed by the availability of suitably positioned restriction sites. Here we describe the basis for homologous recombination cloning in E. coli, the available tools and resources, together with a protocol for long range cloning and manipulation of an Arabidopsis thaliana gene locus, to create constructs coordinately driven by locus-specific regulatory elements.
\end{abstract}

\section{Introduction}

Plant bacterial artificial chromosome (BAC) resources are being generated for ever increasing numbers of species, providing scientists with long-range physical maps and associated sequence data for both model and crop plants. This provides opportunities for reverse genetics and functional studies at the level of the gene locus and above. The latter requires methods for the cloning and manipulation of large DNA fragments, without the limitations imposed by the need for suitably positioned restriction enzyme sites. Significant advances in this respect arose from the development of homologous recombination (HR) cloning in Escherichia coli, based on RecE/RecT (ET) $[1,2]$ and $\lambda$ RED operon gene products $[3,4]$. Essentially, in ETbased strategies, PCR-amplified linear DNA fragments with short regions of homology ( $50 \mathrm{bp}$ to $60 \mathrm{bp}$ ) are precisely targeted into any DNA sequence including high copy number plasmids, the E. coli chromosome and BACs. RED-based protocols rely on a defective $\lambda$ prophage to provide functions that protect and recombine the linear DNA fragments, under the control of a temperature sensitive $\lambda$ cl-repressor, with recombinogenic functions switched on at $42^{\circ} \mathrm{C}$ and off at $32^{\circ} \mathrm{C}$. This fixed induction window helps to reduce unwanted rearrangements, allowing DNA to be stably cloned.

HR-cloning in E. coli is widely used in the biomedical research field and is becoming an established tool for BAC engineering in functional genomic studies [5]. Its applications include recombinogenic targeting for gene disruption or replacement and subcloning of BAC DNA by direct isolation of specific genomic regions. A general schematic of HR cloning is given in Fig. 1. Thus, the construction of transgenes for plant functional genomics or the next generation of genetically modified crop plants may benefit from the level of precision engineering offered by HRcloning. 

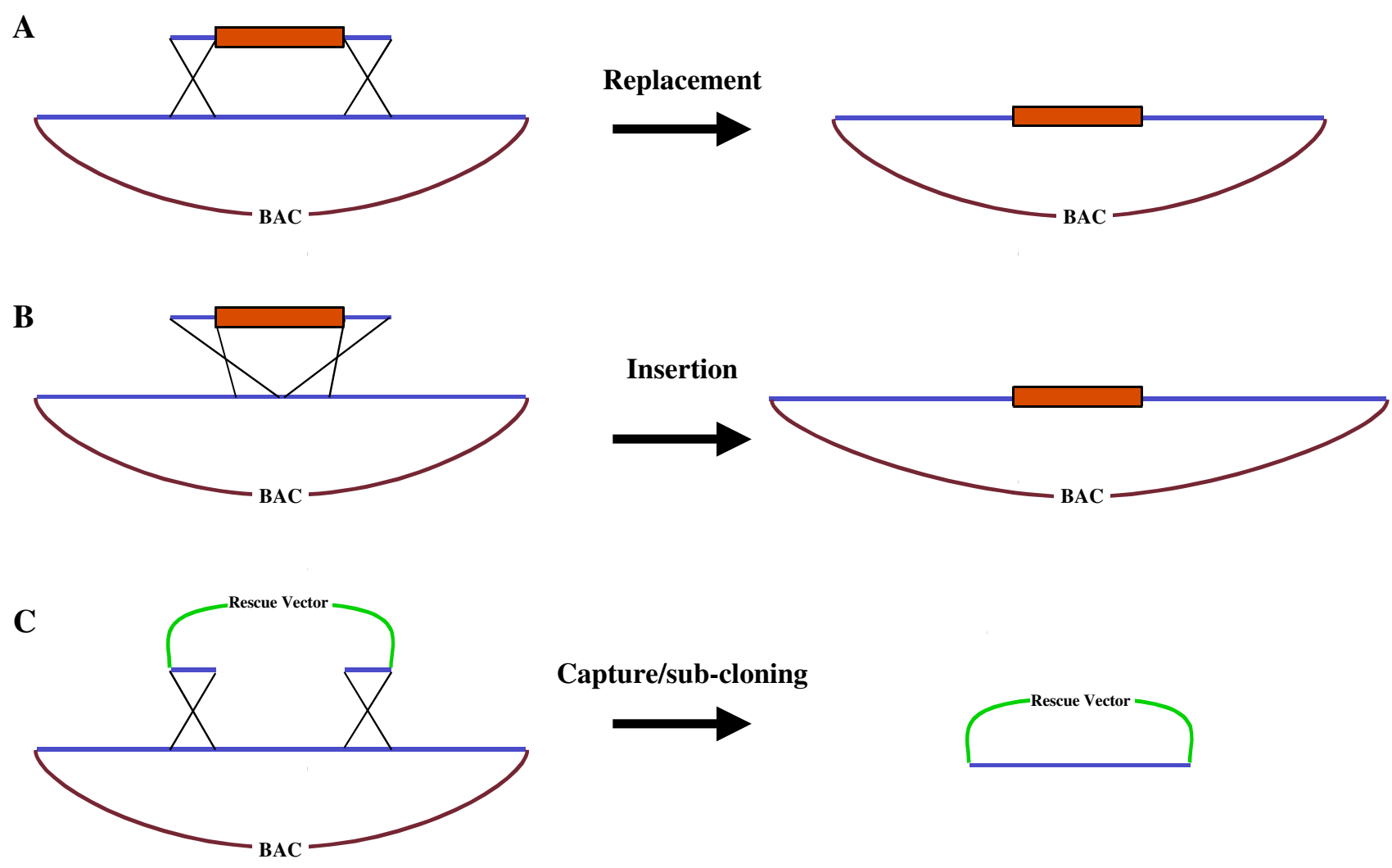

\section{Capture/sub-cloning}

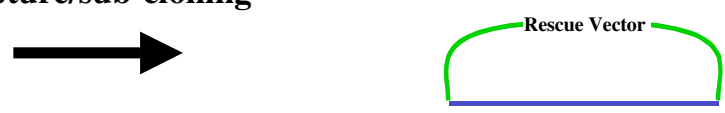

Figure I

Schematic representation of the basic applications of homologous recombination cloning in $E$. coli for genetic engineering. Homologous recombination cloning in $E$. coli can be used for gene replacement (A), insertion (B) or sub-cloning of target sequences into alternative plasmid vectors. The recombination is mediated by linear DNA fragments (usually generated by PCR), including target site-specific homology arms and a counter selectable antibiotic resistance gene marker.

Our interest in long-range HR cloning was driven by a desire to create plant-specific tools and transgene constructs that target expression to the shoot apical meristem. We wanted to express the bean (Phaseolus coccineus) GAPc2ox1 (encoding GA 2-OXIDASE 1, which degrades bioactive gibberellin) in the shoot apex of sugar beet (Beta vulgaris) plants and study the effect on flowering. We present details of our constructs and the molecular tools (plasmids) developed to create these constructs by RED cloning.

\section{Materials \\ Reagents}

- E. coli strain EL250 (genotype DH10B [ $\lambda$ cI857(crobioA) $<>$ ara $\left.-\mathrm{P}_{\mathrm{BAD}} f l p e\right]$ where $<>$ indicates that cro-bioA has been substituted with araC- $\mathrm{P}_{\mathrm{BAD}} f l p e$ ) available from the authors of [3] who have developed a number of different strains including EL350 (with inducible araC- $\mathrm{P}_{\mathrm{BAD}^{-}}$ cre). These strains carry a defective $\lambda$ prophage with red and gam recombination genes under the control of the $\lambda \mathrm{P}_{\mathrm{L}}$ promoter and exo and bet tightly controlled by the temperature sensitive cI857 repressor. Exo and Beta provide recombinogenic function while Gam inhibits the E. coli RecBCD nuclease from degrading electroprated linear DNA fragments. The promoter of the araBAD operon $\left(\mathrm{P}_{\mathrm{BAD}}\right)$ is induced by L-arabinose for flpe and cre expression enabling removal of sequences between FRT and LoxP sites respectively. We used EL250 to enable removal of the kanamycin gene in our FRT-mPGK-Tn5-neo-FRT cassette. OUR RESULTS: The marker gene was removed as described [3] and worked with 90\%-100\% efficiency and we were able to recover $100 \mathrm{~s}$ of colonies which had become kanamycin sensitive.

- Luria Bertani (LB) broth and plates supplemented with antibiotics as required 
- Fully sequenced BAC, PAC or other clones with desired gene locus. Plant BAC and PAC clones are widely available from a number of different sources, including individual labs and organisations e.g. The Arabidopsis Biological Resource Centre (ABRC) http://www.biosci.ohiostate.edu/ plantbio/Facilities/abrc/abrchome.htm or the Nottingham Arabidopsis Stock Centre (NASC) at http:// arabidopsis.info/ and, http://www.dna.affrc.go.jp/ for rice genes and others. The AtSTM locus used in our experiments is cloned in BAC F24o1, sourced from the Arabidopsis Biological Resource Centre, Columbus, Ohio.

- pUC-based vectors to be used for making (i) the locus rescue gap-repair construct (must be counter selectable to the BAC/PAC), and (ii) the gene of interest (GOI) targeting cassette construct (must contain a counter selectable marker to the gap-repair construct).

- High fidelity Taq DNA polymerase. Preferably one which retains A-tails for TA cloning, e.g. the Expand High Fidelity PCR system (Roche Diagnostics).

- PCR primers - four locus-specific primers to amplify DNA fragments at the locus border flanks for the gaprepair rescue construct and two target site-specific primers (minimum 70 bp long) to generate GOI targeting products with destination site specific 5' and 3' homology arms.

- PCR product and gel purification kits e.g. the Qiagen QIAquick $^{\mathrm{TM}}$ range and DpnI restriction enzyme - used to remove plasmid templates from PCR reactions because it only cleaves methylated sites.

- General reagents for standard gene cloning and gel electrophoresis

\section{Equipment}

- Orbital shaking incubator

- Orbital shaking water bath e.g. Grant OLS 200 - essential for induction of recombination functions in bacterial cells.

- Electroporator e.g. Bio-Rad E. coli Pulser

- PCR Machine

- Long wave UV transilluminator - long wave ultra violet light is less damaging to DNA during excision of bands from gels. UV-damaged DNA will not recombine efficiently.

- Electrophoresis equipment capable of field inversion gel electrophoresis (FIGE) or pulsed field gel electrophoresis
(PFGE) e.g. BioRad CHEF DR-II, DR-III or Mapper ${ }^{\mathrm{TM}}$ XA, for efficient resolution of large DNA fragments

- Spectrophotometer for cell density quantification

- Temperature controlled centrifuge able to run at $4^{\circ} \mathrm{C}$

\section{Protocol}

The protocols outlined below describe the development of (i) an AtSTM-locus specific gap-repair rescue vector, (ii) a plant gene targeting construct with a removable kanamycin resistance marker cassette from pGK-FRT [6], under the control of both the bacterial Tn 5 promoter and the mouse phosphoglycerate kinase (mPGK) promoter for selection in prokaryotes and eukaryotes respectively. This provides templates for PCR amplification of selectable gene fragments that can be precisely targeted into any desired gene locus; and (iii) a bean (Phaseolus coccineus) GAPc2ox1 transformation construct, co-ordinately driven by "all" AtSTM locus elements, designated pSTM17::GAPC2ox1. We have also constructed a pENTR4based AtSTM gap-repair rescue vector for the production of a Gateway ${ }^{\mathrm{TM}}$ (Invitrogen) compatible entry clone and generic T-DNA transformation constructs as well as an $m g f p 5-E R$ targeting cassette. The pSTM17::GAPc2ox1 was successfully transformed into sugar beet, demonstrating for the first time that the mouse PGK promoter is fully functional in transgenic plants, thus enabling the direct exploitation of existing mammalian tools.

\section{Key steps in the EL250 RED-HR locus rescue and engineering procedure}

1. Design of PCR primers for amplification of locus rescue (retrieval) homology arms and also for GOI targeting

\section{Construction of a gap-repair locus rescue vector.}

3. Construction of a targeting vector containing the GOI upstream of a counter selectable marker (different from that in the gap-repair construct).

4. Electroporation of EL250 cells with the BAC or clone containing the desired gene locus and preparation of electrocompetent BAC/EL250 cells induced for Exo, Beta and Gam functions.

5. Performance of gap-repair locus rescue, in cells treated as above; selection of recombinants and confirmation by restriction digestion analysis and sequencing. Transformation of the rescued locus plasmid into fresh EL250 cells.

6. PCR amplification, purification and quantification of the GOI targeting cassette and its site-specific recombination into the rescued locus plasmid in EL250 
A

BAC F24o1 (GenBank No. AC003113) 86372 bp

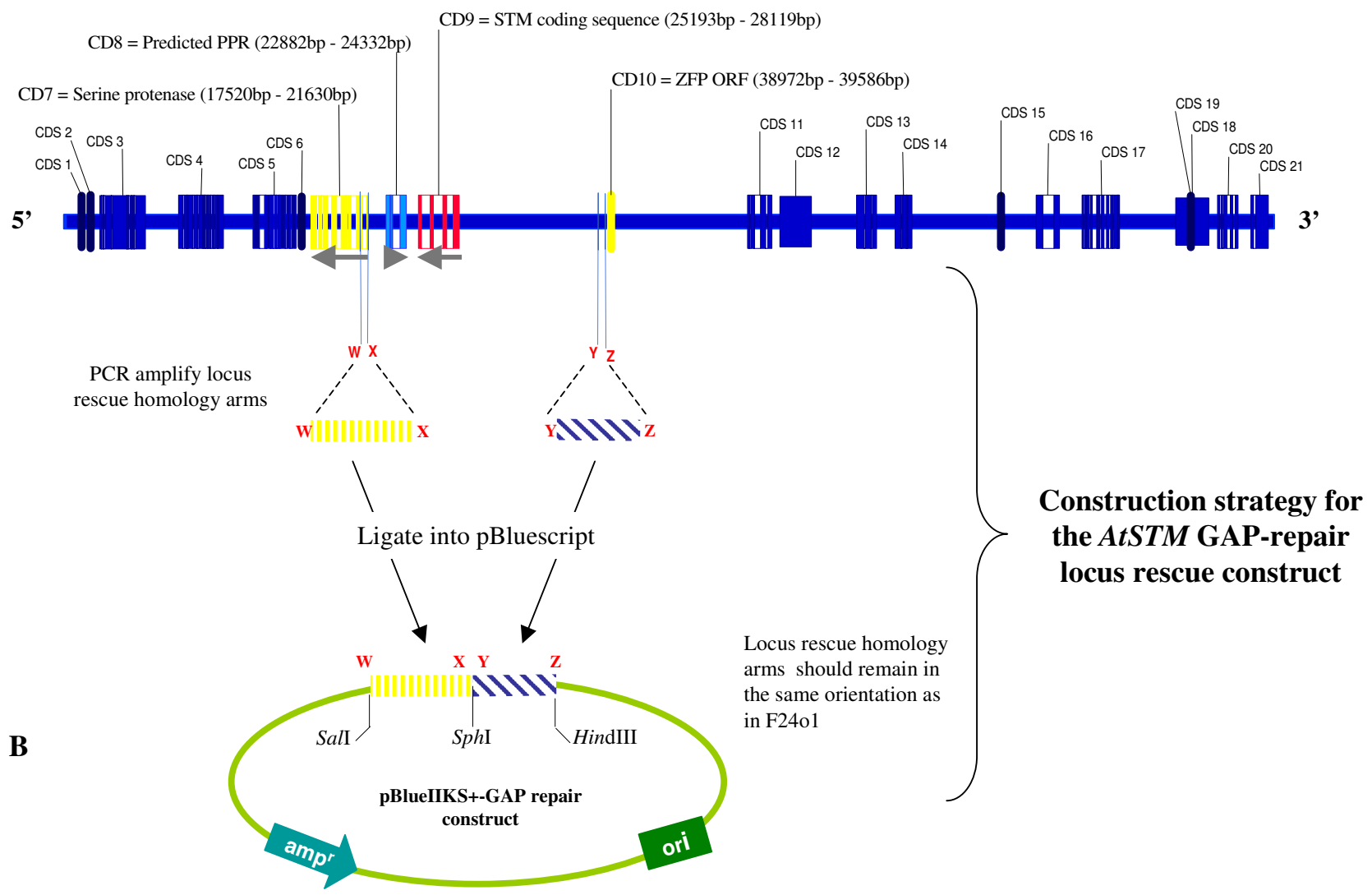

\section{Figure 2}

Maps of the Arabidopsis BAC F24o I and the AtSTM gap-repair construct. A: A physical map of BAC F24ol, showing the relative positions of the 2 I coding sequences (CDS), including STM (CDS 9) and its immediate neighbours (CDS 7, 8 and $10)$ which, are illustrated in different colours and greater detail to show the exon blocks making up each open reading frame. Grey arrows show the orientations of the serine protease, predicted pentatricopeptide repeat protein (PPR), SHOOTMERISTEMLESS (STM) and Zinc Finger Protein (ZFP) open reading frames. B: The gap-repair construct and a schematic representation of the basic protocol used to generate the AtSTM downstream (yellow vertical dashed line: $W$ - X) and upstream (blue diagonal dashed line: $Y-Z$ ) homology arms to create it in the pBluescriptll KS+ vector backbone. W, X, Y and Z are the PCR primers used to generate each homology arm fragment The Sall, Sphl, and Hindlll cloning sites were incorporated into the PCR primers, the sequences of which are given in Additional file I.

cells. Selection and confirmation of recombinants as above.

The recombineered plasmid is now ready for application in functional analyses as desired.

\section{Primer design and plasmid constructs}

Primers

Primer sequences for the AtSTM HR rescue protocol described here are given in Additional file 1. Careful atten- tion must be paid to the design of primers for generating locus rescue (LR) homology arms (HA) to ensure that their orientation in the resultant gap-repair vector is correct for DNA double stranded break repair homologus recombination. A total of four short (18 to $20 \mathrm{bp}$ ) primers will be required and can if necessary, include restriction sites to enable cloning into the gap-repair vector so that the gap-repair construct can be linearised between the LRHAs. Fig. 2A shows our AtSTM gap-repair construct. 
For the GOI targeting cassette, primers must include at least 30 to $50 \mathrm{bp}$ at the $5^{\prime}$ end, to provide homology arms for site-specific recombination. The target site sequence must not have any mismatches as this will inhibit recombination. It is therefore essential to source primers from suppliers able to guarantee sequences of long primers. Our primers were custom made by Sigma Genosys.

\section{Plasmid constructs}

IN OUR HANDS: Creation of these basic plasmids was the key limiting step as it is dependent on conventional cloning and therefore, on the availability of suitably placed restriction sites. However, once constructed, the gene targeting constructs can be used to target the expression cassette into any desired site whereas the gap-repair construct is suitable only for subcloning the specific gene fragment/locus. Our targeting construct backbone has therefore been designed to include a plant-specific polyA signal (Nopaline synthase (nos) termination sequence), for generic use with any plant cDNA sequence.

\section{AtSTM gap-repair construct}

Using BAC F24o1 DNA template (represented in Fig. 2A), and the Expand High Fidelity DNA polymerase PCR system (Roche Diagnostics) we amplified 564 bp (incorporating 5'SalI and 3'SphI sites) and 479 bp (incorporating 5'SphI and 3'HindIII sites) homology arms respectively at the downstream and upstream flanks of the AtSTM locus (Fig. 2B). At the start of our project, the pentatricopeptide repeat protein (PPR) downstream of the STM coding sequence was annotated as a predicted ORF and we therefore opted to include it in the STM locus fragment. Now, it would be excluded as the locus boundary. PCR reactions included primers HindIII 3' hyp/SphI 5' hyp or Sprot H1 Sali/Sprot H1 SphI at $0.3 \mu \mathrm{M}$ each and were incubated for 1 cycle at $94^{\circ} \mathrm{C}$ for $2 \mathrm{~min}$. followed by 30 cycles of $94^{\circ} \mathrm{C}$ for $15 \mathrm{sec} ; 64^{\circ} \mathrm{C}$ for $30 \mathrm{sec} ; 72^{\circ} \mathrm{C}$ for $1.5 \mathrm{~min}$; and 1 cycle of $72^{\circ} \mathrm{C}$ for $5 \mathrm{~min}$. The individual products were then subcloned into pGEM-T Easy (Promega), recovered and cloned into pBluescript II SK+ (Stratagene) in a three-way ligation reaction, to create the gap-repair construct.

NOTE: In our hands, cloning of PCR products is more efficient if we shuttle them via a PCR cloning vector. Any TA cloning vector is suitable. In this case, it is important to ensure that the proof reading activity of the Taq Polymerase used does not remove A-tails.

\section{Targeting construct backbone}

The $1.8 \mathrm{~kb}$ FRT-mPGK-Tn5-neo-FRT cassette was PCR amplified with SacII in the $5^{\prime}$ primer (PGK-FRT upper) and ApaI in the 3' primer (PGK-FRT lower) from pPGKFRT (obtained under a Material Transfer Agreement from Dr Francis Stewart, EMBL, Heidelberg - now at University of Technology, Dresden) and cloned into SacII/ApaI clon- ing sites downstream of the Green Fluorescent Protein (GFP) gene of polyGFP3 (a kind gift from Dr E. Amaya, Gurdon Institute, Cambridge). The GFP gene was then replaced with the Nopaline synthase Terminator (NosTer) from pAL69 (pFC6 with NosTer in the multiple cloning site. A kind gift from Dr Dave Lonesdale at the John Innes Centre, Norwich, UK), to create the basic plant gene targeting vector pNosTerFRT-neo (Fig. 3A), which can be used to receive any GOI.

\section{GAPc2ox targeting construct}

The full length GAPc2ox1 cDNA in plasmid pST33 (a kind gift from Drs Andy Philips and Peter Hedden, Rothamsted Research) was excised with Spel/XhoI and cloned into the NheI/SalI site of pNosTerFRT-neo (Fig. 3Bi).

\section{mgfp5-ER targeting cassette}

The mgfp5-ER gene (GenBank U87974) was isolated from pBIN35S-mgfp5-ER (a kind gift from Dr Jim Haseloff, Department of Plant Sciences, University of Cambridge, $\mathrm{UK}$ ) as a BamHI/SacI fragment and cloned into the SmaI site of pNosTerFRT-neo (Fig. 3Bii)

WARNING! GUS reporter cassettes are not suitable as they are able to recombine with the endogenous (chromosomal) E. coli gene during HR cloning.

\section{RED Cloning Protocol}

Original methods and information on how to obtain host cells can be found at the recombineering website http:// recombineering.ncifcrf.gov/

\section{A: Preparation of electrocompetent EL250 cells}

These cells will be periodically used to receive new plasmids as they are constructed and required for recombineering. It is therefore advisable to make and store a sizable batch.

1. Streak out cells on LB plates and grow at $32^{\circ} \mathrm{C}$. The cells are temperature sensitive and will die at $37^{\circ} \mathrm{C}$

2. Inoculate a single colony into $5 \mathrm{ml} \mathrm{LB}$ and grow overnight.

3. Inoculate $1 \mathrm{ml}$ of the overnight culture into $50 \mathrm{ml} \mathrm{LB}$ in a $500 \mathrm{ml}$ flask and grow at $32{ }^{\circ} \mathrm{C}$ with shaking at $200 \mathrm{rev}$ olutions per minute (rpm) until the cells density has reached $\mathrm{OD}_{600}=0.5-0.8$.

4. Spin cells at $4{ }^{\circ} \mathrm{C}$ (rotor must be pre-cooled) and wash with $5 \mathrm{ml}$ ice cold sterile distilled water. Spin and discard supernatant. Repeat wash with $5 \mathrm{ml}$ aliquots of ice cold SDW two more times. 


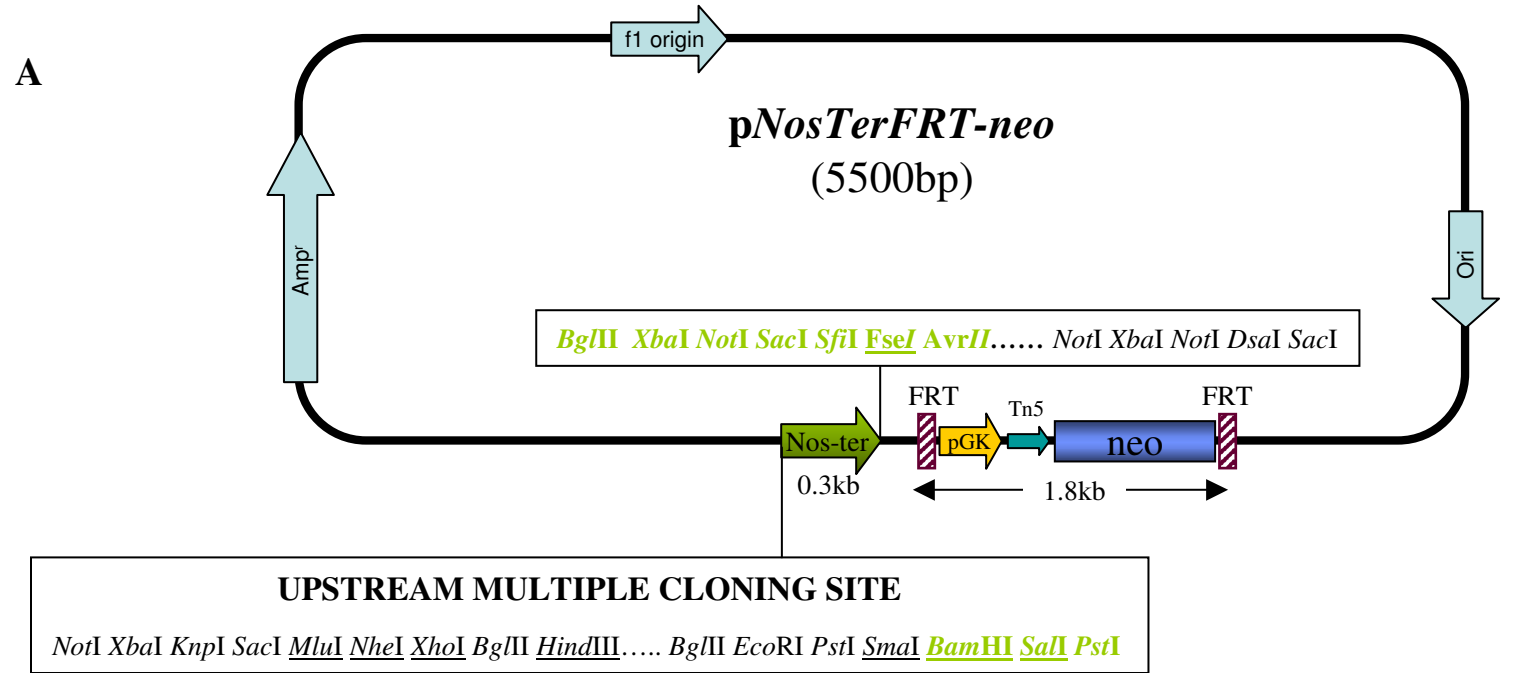

B TARGETING CONSTRUCT

(i)

(ii)
pGAPc2oxlFRT-neo

EcoRI

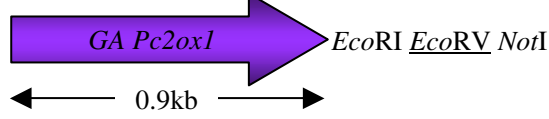

pmgfp5-ERFRT-neo

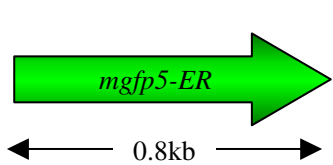

SpeI/XhoI fragment from pST33 was cloned into pNosTerFRTNeo cut with NheI/SalI

Bam $\mathrm{HI} / \mathrm{SacI}$ fragment from pBin-mgfp5-ER was blunt end ligated into pNosTerFRT-Neo cut with SmaI

Figure 3

Map of the targeting plasmid backbone pNosTerFRT-neo and GAPc2ox I/mgfp5-ER targeting cassettes. A: Structure of the targeting plasmid backbone pNosTerFRT-neo, showing the relative positions of the Nopaline synthase polyA signal sequence (NosTer), the FRT-mPGK-Tn5-neo-FRT selection cassette and the restriction sites mapped up and downstream of the NosTer sequence. Upstream sites may be used for cloning any GOI cDNAs to create targeting constructs. Sites shown in green were introduced with the NosTer fragment; all other sites originate from PolyGFP3. Unique sites are underlined. B: Representations of $\mathrm{GO}$ sequences with available upstream and downstream restriction sites as appropriate, together with the cloning strategies employed to clone them into pNosTerFRT-neo to make the targeting cassette constructs.

5. Finally resuspend cells in $500 \mu \mathrm{l}$ of ice cold sterile distilled water and aliquot $100 \mu \mathrm{l}$ lots into cooled $1.5 \mathrm{ml}$ microfuge tubes.

NOTE: Cells can be used immediately or re-suspended in ice cold sterile $10 \%(v / v)$ glycerol and stored at $-80^{\circ} \mathrm{C}$ until required.

\section{B: Transformation of BAC F24ol and induction of recombinogenic} function in EL250

1. On ice, add $10 \mathrm{ng}-100 \mathrm{ng}$ of F24o1 DNA to $50 \mu \mathrm{l}$ of competent EL250 cells. Mix by gentle pipetting and transfer to a pre-cooled $0.1 \mathrm{~cm}$ electroporation cuvette.
2. Pulse at $1.75 \mathrm{kV}$ in a Bio-Rad E. coli Gene Pulser. Immediately add $1 \mathrm{ml} \mathrm{LB}$ broth and incubate at $32{ }^{\circ} \mathrm{C}$ for $1-1.5$ $\mathrm{h}$ in a shaking incubator set at $200 \mathrm{rpm}$

3. Plate cells on LB kanamycin and select for transformants. NOTE: It is advisable at this stage to check the integrity of the BAC clone by restriction digestion analysis, to ensure that there have been no rearrangements.

4. Grow F24o1/EL250 cells as described in A: steps 1 - 3 except that all LB media must be supplemented with kanamycin (or relevant antibiotic) to select for the BAC. NOTE: before the next step, ensure that the shaking water bath 
is switched on early and stabilised at $42^{\circ} \mathrm{C}$ ready for use and pre-warm the conical flask. An ice slurry bath must also be made ready - DO NOT USE JUST ICE - it will not cool cells fast enough.

5. For induction, transfer $10 \mathrm{ml}$ of the growing culture into a pre-warmed $250 \mathrm{ml}$ conical flask and incubate in the water bath at $42{ }^{\circ} \mathrm{C}$ with shaking at $200 \mathrm{rpm}$ for a total of $15 \mathrm{~min}$. NOTE: Keep the remaining $40 \mathrm{ml}$ of culture at 32 ${ }^{\circ} \mathrm{C}$ to act as a non-induced control.

6. Immediately after $15 \mathrm{~min}$. place the flask in the ice slurry bath and swirl by hand to quickly cool down the cells. Include a similar flask with $10 \mathrm{ml}$ of non-induced control cells - this will be cooled down and treated in the same way as the test cells from now on. NOTE: (i) Induced cells must be used immediately as they will lose activity above $0^{\circ} \mathrm{C}$. Therefore it is important to work quickly from now on. However, cells may be kept on ice for a total of $40 \mathrm{~min}$. without significant loss of activity. (ii) Ensure that the centrifuge and rotor are pre-cooled to $4{ }^{\circ} \mathrm{C}$ before the next step.

7. Centrifuge the $10 \mathrm{ml}$ aliquots of induced and control cells for $8 \mathrm{~min}$ at $5500 \mathrm{~g}$ and at $4^{\circ} \mathrm{C}$. Retrieve pellets and wash three times in $1 \mathrm{ml}$ ice cold sterile distilled water and centrifuge as above. NOTE: To save time, washing steps can be carried out in $1.5 \mathrm{ml}$ microfuge tubes keeping everything ice cold and centrifuging at $4{ }^{\circ} \mathrm{C}$ for 20 seconds each time.

8. After final wash, re-suspend the cell pellet in $100 \mu \mathrm{l}$ of ice cold sterile distilled water. This is enough for two electroporation transformation reactions.

\section{C: AtSTM locus rescue from BAC F24ol by gap-repair HR}

Before starting: Ensure that purified and linearised gaprepair vector is available at concentrations suitable to deliver 10 to $100 \mathrm{ng}$ in volumes up to $10 \mu \mathrm{l}$. We strongly recommend gel quantification with known standards as we find this more accurate than $\mathrm{OD}_{260 \mathrm{~nm}}$ measurements.

N.B. All HR experiments should be carried out with the induced and un-induced control cells in parallel.

1. Using linearised gap-repair construct DNA, electroporate induced competent F24o1/EL250 cells as described in B: steps $1-2$.

2. Select recombinants on LB supplemented with antibiotic marker for the gap-repair vector. We used pBluescriptII KS+ and therefore selected on LB ampicillin. The use of pBluescript also limits the size of insert which can be rescued and $17 \mathrm{~kb}$ was the largest fragment we were able to retrieve by gap repair HR cloning.
3. Recover recombinant plasmids and confirm correct recombination events by restriction digestion analysis and sequencing. This is important since incorrect events may still be selectable with the antibiotic marker. OUR RESULTS: The number of colonies recovered was typically small $(2-4)$ but of these, 50\% were correct. The remainder were the result of illegitimate recombination events. See Fig. 4 for our HR strategy and the result of our AtSTM gap-repair rescue to give the plasmid pBlueAtSTM17. NOTE: Because of the large DNA fragments involved, it is advisable to use Field Inversion Gel Electrophoresis or Pulsed Field Gel Electrophoresis as appropriate for clarity in resolution.

TROUBLE SHOOTING: Growth of un-induced colonies on selective plates suggests incomplete digestion of the gap-repair construct during linearization. However, the number of colonies should be low (we typically recovered 5 - 10 colonies from un-induced cells). Otherwise repeat with improved digestion and/or gel purification of the linearised gap repair construct.

4. Transform the rescued plasmid into fresh EL250 cells and prepare induced competent cells as described, ready for the locus targeting experiment. We designated these cells STM17/EL250 because they contained rescued $\sim 17.5$ kb of the AtSTM locus. NOTE: For our application, we use a protoplast based direct transformation method and therefore opted to use pBluescript as the backbone for our gap repair and eventual transformation construct. However, for Agrobacterium-based systems, we recommend using a Gateway compatible Entry vector (available from Invtrogen: http:// www.invitrogen.com) as this will enable subsequent transfer of the captured, manipulated locus into a T-DNA binary destination vector for example the ones available from Plant Systems Biology (VIB-Gent University: http://www.psb.rug.ac.be/ gateway) or the pEarlyGates vectors, details of which can be found at the website: http://www.biology.wustl.edu/ pikaard/pEarleyGate\%20plasmid\%20vectors/

Table\%20of\%20vectors.html.

Recently, we have successfully created an AtSTM locus rescue vector based on the Invitrogen pENTR4 Gateway ${ }^{\mathrm{TM}}$ compatible vector in which we plan to capture/manipulate the locus as described and determine the success rate of transfer into a promoterless T-DNA destination vector pB7WG2 335 S (based on pB7WG2 from VIB-Gent University). These are newly available resources that should enable the creation of constructs for the more generic Agrobacterium-mediated plant transformation systems.

WARNING!: Direct use of T-DNA vectors as gap-repair constructs in RED cloning although attractive, may prove problematic because of the common use of a limited number of identical or very similar promoter and polyA signal sequences, which if also present in the targeting 


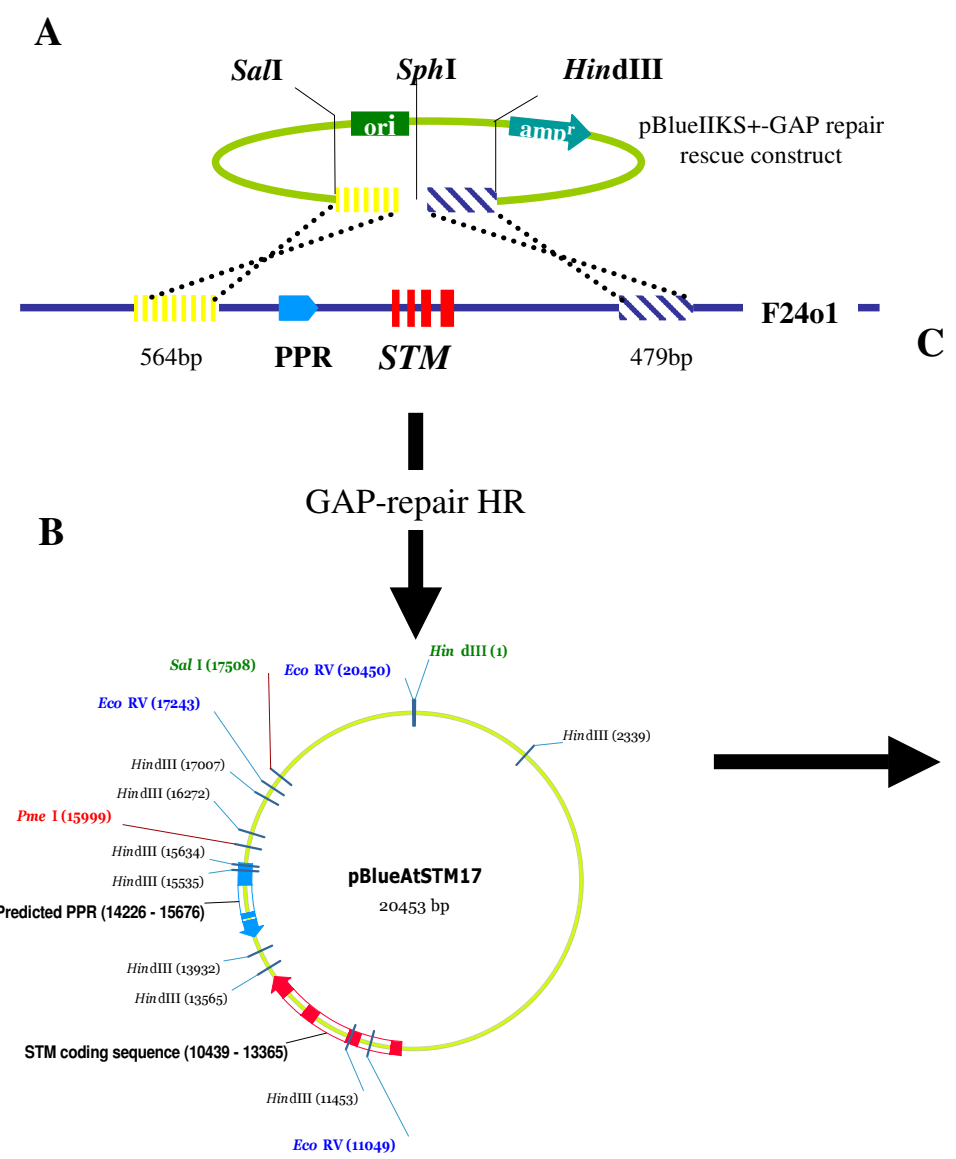
Restriction digestion analysis of independent GAP
repair recombinations into the pBlueIIKS+ construct.
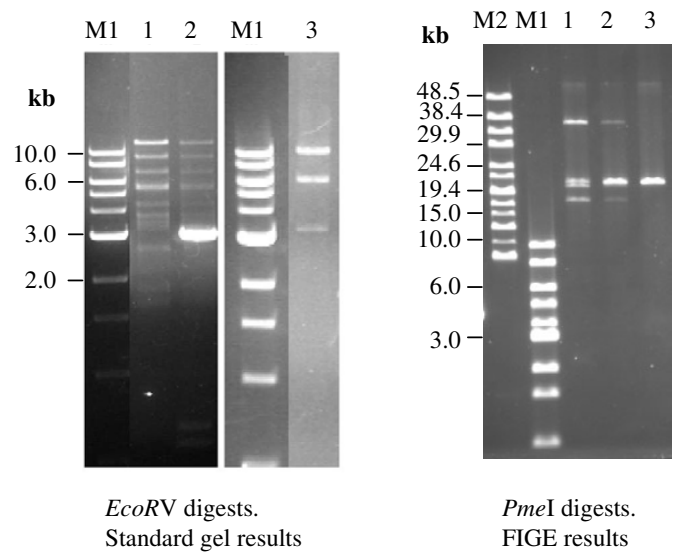

Lane 3 shows the restriction patterns as expected from the correct recombination event in pBlueAtSTM17 as in B

\section{Figure 4}

gap repair HR rescue of the AtSTMlocus. Schematic representation of the HR rescue of the AtSTM locus into the pBluescript gap repair vector $(A)$ and the resultant pBlueAtSTMI7 construct as expected from the correct recombination event. Diagnostic EcoRV sites are shown in blue, Pmel site in red, together with their co-ordinates within pBlueAtSTMI7. Sall and HindIII sites originally engineered in the homology arms of the gap repair vector are shown in green. (B). The results of EcoRV and Pmel restriction digestion of three plasmids resulting from independent recombination events from a single experiment, showing that only the plasmid in lane 3 resulted from the correct recombination. $\mathrm{MI}=\mathrm{I} \mathrm{kb}$ ladder (New England Biolabs); $\mathrm{M} 2=$ High Molecular Weight Marker (Invitrogen).

cassette will result in illegitimate recombination events. For this reason, we did not attempt any experiments with T-DNA vectors, opting instead to go via the Gateway ${ }^{\mathrm{TM}}$ system as detailed above.

\section{D: Replacement of AtSTM exon I by in-frame fusion of the promotorless GAPc2ox-FRT-neo-FRT targeting cassette}

Before starting: The purified, DpnI treated and quantified PCR amplified GOI targeting cassette should be made ready for this experiment.

1. Using up to $100 \mathrm{ng}$ of the PCR amplified GAPc2ox1FRT-neo-FRT targeting cassette, electroporate induced
STM17/EL250 cells as described in B: steps $1-2$. The targeting cassette was amplified with HotStar Taq DNA polymerase (Qiagen) and primers 28001 frtlow and 2oxexon $1(0.3 \mu \mathrm{M}$ each $)$ in a $50 \mu \mathrm{l}$ reaction volume. Incubation conditions were 1 cycle $95^{\circ} \mathrm{C}$ for $1 \mathrm{~min}$ followed by 20 cycles of $94^{\circ} \mathrm{C}$ for $15 \mathrm{sec} ; 68^{\circ} \mathrm{C}$ for $4.5 \mathrm{~min}$. (with a 5 sec. time increment in each cycle); followed by 1 cycle of $68^{\circ} \mathrm{C}$ for $10 \mathrm{~min}$.

2. Select recombinants on LB kanamycin. Recover plasmids and confirm by restriction digestion analysis and sequencing. Plasmids are now ready for application in functional assays. OUR RESULTS: We recovered many 
A

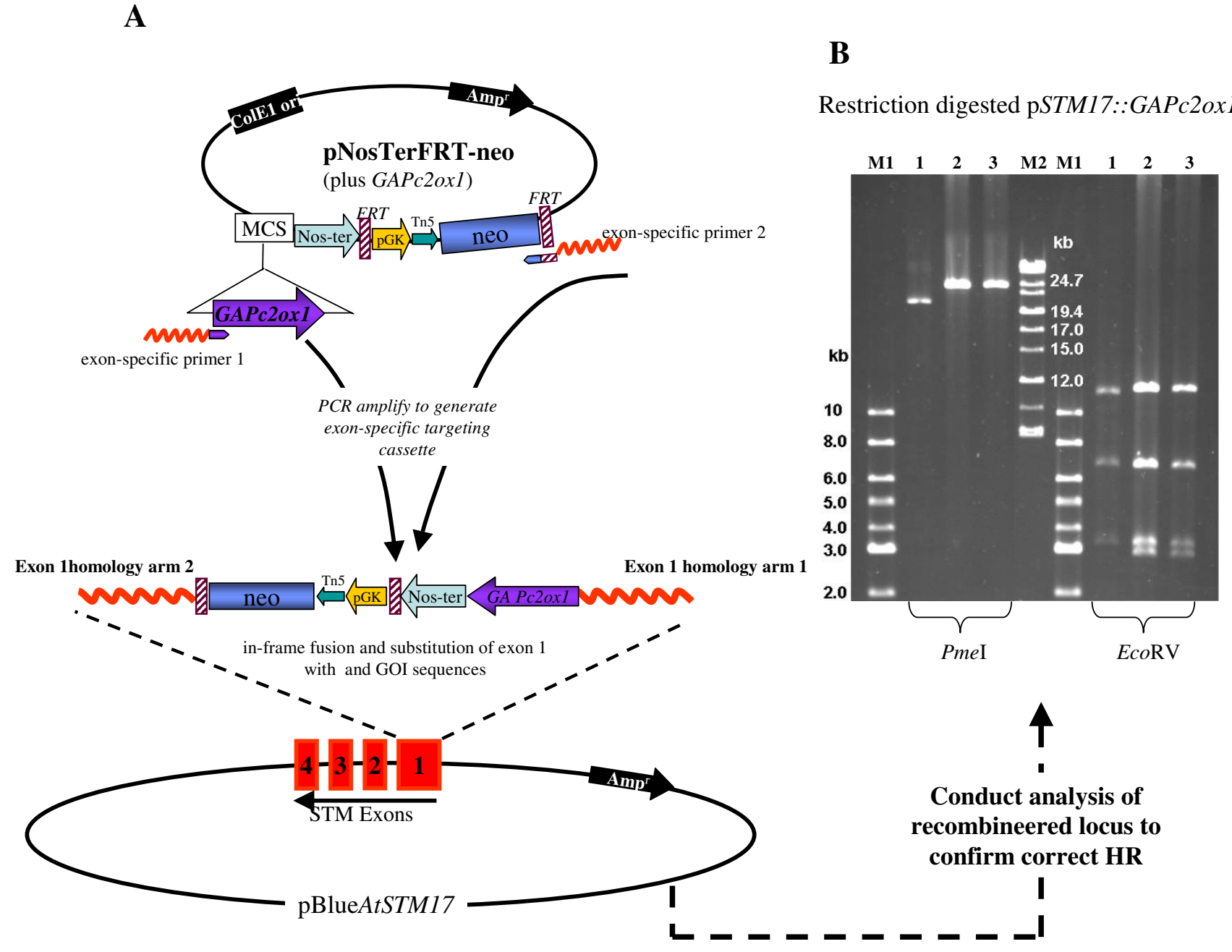

Figure 5

Replacement of AtSTM exon I by in-frame fusion of GAPc2ox I. A: Schematic representation of the strategy used to generate the GAPC2ox I cassette from pNosTerFRT-neo (plus GAPc2oxl) plasmid and target it into the AtSTM locus (captured in PBlueAtSTMI7) by in-frame substitution of exon I sequences. B: FIGE gel results of Pmel and EcoRV digested

PSTM I 7::GAPc2ox I from two independent recombination events (lanes 2 and 3), clearly showing the increased size of the linearised construct (Pmel digest) and the additional fragment (EcoRV digest) due to the recombination of the GAPc2ox I cassette. Lane I shows the control result with the original pBlueAtSTM I 7. MI = I kb ladder (New Endgland Biolabs); M2 = High Molecular Weight Marker (Invitrogen).

colonies at this stage (100 s), of which 6\% were correct by restriction digestion analysis and sequencing. For example, we typically screened between 30 and 35 colonies from which two were correct. See Fig. 5 for the results of our GOI targeting experiment. NOTE: It is advisable at this stage to at least sequence across the recombination site into the GOI cassette to confirm the integrity of the gene cassette before proceeding with functional assays in transgenic plants.

TROUBLE SHOOTING: High un-induced colony numbers on selective plates suggest targeting cassette template contamination instead of recombination. Check DpnI digests and use this in combination with gel purification to remove template DNA from the target cassette PCR product prior to electoporating for HR. In our experience, if the number of colonies from un-induced cells is at least 50 - 100 fold less than from the induced cells, then it was worth screening colonies from induced cells.

Our final recombineered construct was designated AtSTM17:: GAPc2ox1 and was transformed into sugar beet guard cell protoplasts [7] from which we successfully 


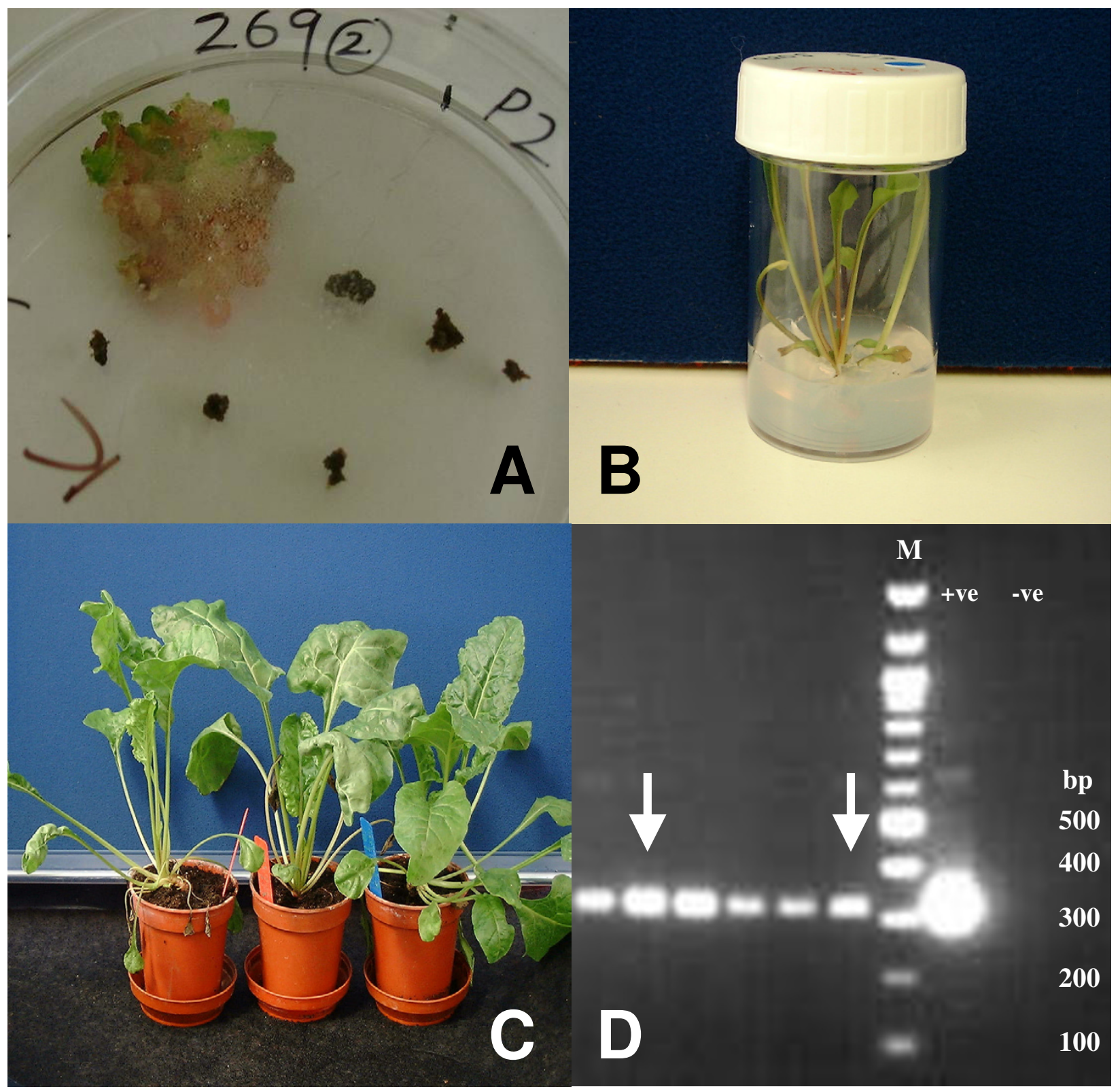

Figure 6

Kanamycin selection and regeneration of transgenic sugar beet guard cells transformed with the

PSTMI 7::GAPc20xI constructs containing the neomycin gene driven by the mouse PGK promoter. A: Transgenic sugar beet callus grown from transformed sugar beet guard cell protoplasts cultured on medium supplemented with kanamycin at $100 \mu \mathrm{g} \mathrm{ml}-1$, clearly showing a difference between the healthy (green) callus and the dead (brown) non-transgenic callus. B: Shoots regenerated from transgenic sugar beet callus as above. C: Resultant transgenic seedlings in compost. D: Agarose gel showing the results of neomycin gene-specific PCR conducted on genomic DNA template extracted from sugar beets generated from two independent transformation events with PSTM I 7::GAPc2ox I including the kanamycin driven by the mouse PGK promoter (arrows) compared with individuals from lines transformed with constructs containing a kanamycin cassette driven the CaMV35S promoter (the remaining PCR product bands). Sequences from other regions of the PSTM I 7::GAPc2ox I construct were also detected by PCR and Southern blot hybridisation (results not shown). +ve = DNA plasmid template control, -ve = DNA template-free control; $M=$ New England Biolabs 100 bp ladder. Diagnostic PCR for the neomycin gene sequences was performed using the primers Neo-For 5' CAG GAT GAT CTG GAC GAA GA $3^{\prime}\left(\mathrm{Tm}=57.3^{\circ} \mathrm{C}\right)$ and Neo-Rev $5^{\prime}$ AAG AAG GCG ATA GAA GGC GA $3^{\prime}\left(\mathrm{Tm}=57.3^{\circ} \mathrm{C}\right.$ ). The reactions (Qiagen Master Mix) contained $10 \mu \mathrm{M}$ of each primer, and 50-100 ng genomic DNA template and were incubated at $94^{\circ} \mathrm{C}$ for $3 \mathrm{~min}$, followed by 30 cycles of $94^{\circ} \mathrm{C}$ for 15 sec.; $55^{\circ} \mathrm{C}$ for 30 sec.; $72^{\circ} \mathrm{C}$ for I min and one cycle of $72{ }^{\circ} \mathrm{C}$ for $2 \mathrm{~min}$. 
selected transgenic callus and shoots under kanamycin selection driven by the mouse PGK promoter in the FRTmPGK-Tn5-neo-FRT cassette (Fig. 6). We are now in the process of conducting phenotypic analyses of our AtSTM17:: GAPc2ox1 plants.

\section{Comments}

Manipulation of large DNA fragments to make complex constructs for functional genomics or genetic engineering for crop improvement is possible using HR cloning in $E$. coli. We have successfully used HR cloning in E. coli to subclone the Arabidopsis thaliana SHOOTMERISTEMLESS (STM) gene locus from a BAC clone into pBluescript and to replace exon 1 sequences with a Gibberellin 2-oxidase cDNA gene-of-interest cassette tightly linked to an FRTflanked kanamycin selection marker gene. This cassette is of generic use because firstly, it can be targeted/recombineered into any locus/destination site. Secondly, the kanamycin resistance gene is under the control of both the bacterial Tn5 promoter and the mouse phosphoglycerate kinase promoter (mPGK), which respectively allow for selection in prokaryotes and eukaryotes. We have now demonstrated the utility of the mPGK promoter for driving expression in transgenic plants and this suggests that there may well be increased scope for plant scientists to directly benefit from existing molecular genetic tools developed for application in the biomedical field.

E. coli ET- and RED-HR cloning are well established technologies within the biomedical field and they have many uses besides the creation of transformation constructs with long-range regulatory elements. The identification of regulatory elements or locus control regions located at a distance from the gene sequence can be assisted by this strategy. Point mutations, deletions or insertions, gene fusions and antisense constructs can be engineered on any BAC for functional genomics studies. The scope for plant science is further enhanced by the recently reported application of HR to convert BACs into binary vectors [8] together with (i) the availability of a BAC-based physical map of $A$. thaliana, (ii) freely available genome sequence information through the Arabidopsis Genome Initiative, (iii) access to rice sequence data and BAC resources through The Institute for Genomic Research (TIGR) and the Rice Genome Resource Center (RGP).

Resources for RED/ET cloning are available from Neal Copeland and Nancy Jenkins for both profit and nonprofit organisations. Details can be found at the following website: $\quad$ http://recombineering.ncifcrf.gov/ reagent request.asp. The commercial company GeneBridges http://www.genebridges.com/web/company/ index.htm also offers reagents and a DNA engineering service.

\section{Abbreviations}

$\mathrm{BAC}=$ Bacterial artificial chromosome; $\mathrm{bp}=$ base pairs; FIGE = field inversion gel electrophoresis; GA 2ox = gibberellin 2-oxidase; $\mathrm{GFP}=$ gree fluorescent protein; $\mathrm{GOI}=$ gene of interest; HA = homology $\operatorname{arm}(\mathrm{s})$; HR = homologous recombination; LB = Luria Bertani medium; LR = Locus rescue; $\mathrm{mPGK}=$ mouse phosphoglycerate kinase promoter; $\mathrm{OD}=$ optical density; $\mathrm{PCR}=$ polymerase chain reaction; $\mathrm{ORF}=$ open reading frame; $\mathrm{rpm}=$ revolutions per minute, $\mathrm{UV}=$ ultraviolet.

\section{Competing interests}

The author(s) declare that they have no competing interests.

\section{Authors' contributions}

ESM-G and BG conceived of the study and participated in its design. ESM-G and LCR drafted the manuscript. LCR participated in the design of the study and carried out most of the experimental. ESM-G directed the work, participated in experimental work. All authors read and approved the final manuscript.

\section{Additional material}

\section{Additional File 1}

PCR Primer Sequences. Details of the primers used isolate and manipulate the AtSTM gene locus by homologous recombination in E. coli EL250 Click here for file

[http://www.biomedcentral.com/content/supplementary/17464811-1-6-S1.doc]

\section{Acknowledgements}

Ann Mathews, Roz Williamson and Sarah Yallop for technical assistance molecular analyses and sugar beet transformation.

The project was funded by the Biotechnology and Biological Sciences Research Council of the UK as part of the ROPA scheme.

\section{References}

I. Zhang Y, Buchholz F, Muyrers JP, Stewart AF: A new logic for DNA engineering using recombination in Escherichia coli. Nat Genet 1998, 20:123-128.

2. Zhang Y, Muyrers JP, Testa G, Stewart AF: DNA cloning by homologous recombination in Escherichia coli. Nat Biotechnol 2000, I 8:1314-1317.

3. Lee EC, Yu D, Martinez de Velasco J, Tessarollo L, Swing DA, Court DL, Jenkins NA, Copeland NG: A highly efficient Escherichia coli-based chromosome engineering system adapted for recombinogenic targeting and subcloning of BAC DNA. Genomics 200I, 73:56-65.

4. Yu D, Ellis HM, Lee EC, Jenkins NA, Copeland NG, Court DL: An efficient recombination system for chromosome engineering in Escherichia coli. Proc Natl Acad Sci U S A 2000, 97:5978-5983.

5. Copeland NG, Jenkins NA, Court DL: Recombineering: a powerful new tool for mouse functional genomics. Nat Rev Genet 200I, 2:769-779. 
6. Angrand PO, Daigle N, van der Hoeven F, Scholer HR, Stewart AF: Simplified generation of targeting constructs using ET recombination. Nucleic Acids Res 1999, 27:e 16.

7. Hall RD, Riksen-Bruinsma T, Weyens G], Rosquin IJ, Denys PN, Evans IJ, Lathouwers JE, Lefebvre MP, Dunwell JM, van Tunen A, Krens FA: A high efficiency technique for the generation of transgenic sugar beets from stomatal guard cells. Nat Biotechnol 1996, 14:1133-1138.

8. Takken FL, Van Wijk R, Michielse CB, Houterman PM, Ram AF, Cornelissen $B$ J: $A$ one-step method to convert vectors into binary vectors suited for Agrobacterium-mediated transformation. Curr Genet 2004, 45:242-248.

Publish with Bio Med Central and every scientist can read your work free of charge

"BioMed Central will be the most significant development for disseminating the results of biomedical research in our lifetime. "

Sir Paul Nurse, Cancer Research UK

Your research papers will be:

- available free of charge to the entire biomedical community

- peer reviewed and published immediately upon acceptance

- cited in PubMed and archived on PubMed Central

- yours - you keep the copyright

Submit your manuscript here:

http://www.biomedcentral.com/info/publishing_adv.asp
BioMedcentral 